\title{
The explicit general solution of trivial Monge-Ampère equation
}

Vitaly Ushakov

\begin{abstract}
The general solution of the equation $z_{x x} z_{y y}-z_{x y}^{2}=0$ with minimal smoothness requirements is presented in explicit form; it depends on 2 functions of one variable. In particular, it allows to describe explicitly all developable surfaces (without planar points) in $\mathbb{R}^{3}$. The domain and singularities of the solution are investigated.
\end{abstract}

Mathematics Subject Classification (1991). Primary: 35A10, secondary: 53A05, 35A20, 35B05, 35E15

Keywords. Developable surface, tangent developable, general solution of PDE, initial value Cauchy problem, caustic.

\section{Main Theorem}

The trivial Monge-Ampère equation is

$$
\operatorname{Hess}(z)=z_{x x} z_{y y}-z_{x y}^{2}=0
$$

and sometimes it is called the equation of developable surfaces $[\mathrm{CH}, \mathrm{p} .10]$.

Theorem. The general solution of the equation $\operatorname{Hess}(z)=0$, with the assumption $z_{x x} \neq 0$ everywhere, is given in parametric form:

$$
\left\{\begin{array}{l}
x(u, v)=g(u)-v \cdot f^{\prime}(u) \\
y(u, v)=v \\
z(u, v)=u \cdot g(u)-\int_{0}^{u} g(t) d t+v \cdot\left\{f(u)-u \cdot f^{\prime}(u)\right\}
\end{array}\right.
$$

where $f(u)$ and $g(u)$ are arbitrary functions such that $f \in C^{2}, g \in C^{1}, g^{\prime}(u) \neq 0$ everywhere. Furthermore, the Hesse matrix is

$$
\left(\begin{array}{cc}
z_{x x} & z_{x y} \\
z_{y x} & z_{y y}
\end{array}\right)(u, v)=\frac{1}{g^{\prime}(u)-v \cdot f^{\prime \prime}(u)} \cdot\left(\begin{array}{cc}
1 & f^{\prime}(u) \\
f^{\prime}(u) & {\left[f^{\prime}(u)\right]^{2}}
\end{array}\right) .
$$

I would like to thank Prof. H. Rubinstein and Prof. W. Neumann who kindly afforded me an opportunity to work at the University of Melbourne. 
Since the change of variables $(x, y) \longleftrightarrow(u, v)$ is $C^{1}$ smooth, system (3) gives the following corollary complementing and containing Theorem 1 from [U3].

Corollary. The general solution $z(x, y)$ of the equation $\operatorname{Hess}(z)=0$, with the assumption $z_{x x} \neq 0$ everywhere, is $C^{2}$ smooth, but need not to be $C^{3}$. Moreover, the quotient $z_{x y}: z_{x x}$ is $C^{1}$ and need not to be $C^{2}$.

In Section 3 we shall discuss how to remove the assumption $z_{x x} \neq 0$.

An incomplete solution with $g(u)=u$ and arbitrary $f(u)$ was presented in [HW, pp. 169-170].

Solutions of a similar equation $\operatorname{Hess}(z)=1$ over various domains have been considered in [J1, J2 and SW].

Proof of Theorem. Classical change of variables. The solution of equation (1) can be written explicitly in new coordinates $(u, v)$ which we will now introduce. Let $z(x, y)$ be a solution of equation (1) in the vicinity of the $x$-axis. Then we assign

$$
\left\{\begin{array}{l}
u=z_{x}(x, y) \\
v=y
\end{array}\right.
$$

This new coordinate system has the following geometrical motivation: the $x y$ plane is fibered on rectilinear generators along which the function $z_{x}$ is constant; in the $u v$-plane these generators become the coordinate lines $u=$ const. Or, that is to say, the surface $(x, y, z(x, y))$ is fibered on rectilinear generators along which the tangent plane is constant. More detailed discussion can be found in [U3]. The Jacobi matrix of the change (4) is

$$
\frac{\partial(u, v)}{\partial(x, y)}=\left(\begin{array}{cc}
z_{x x} & z_{x y} \\
0 & 1
\end{array}\right)
$$

thus $\operatorname{det}\left(\frac{\partial(u, v)}{\partial(x, y)}\right)=z_{x x} \neq 0$ and (4) does give a change of variables.

Now our goal is to express $x, y$ and $z$ as functions of $u$ and $v$.

Introduction of function $z_{y}=\boldsymbol{f}\left(z_{x}\right)$. It is not difficult to show that along the rectilinear generators not only the function $z_{x}(x, y)$ but also the function $z_{y}(x, y)$ is constant (see [U3]). Thus, $z_{y}$ depends only on the value of $z_{x}=u$, does not depend on $v$, and we can introduce a new function $f$ :

$$
z_{y}=f(u)
$$

Then from (4) and (5) one can get

$$
u_{y}=\left(z_{x y}=z_{y x}\right)=f^{\prime}(u) \cdot u_{x} .
$$

In this equation the dependent variable is $u(x, y)$. We shall transform the equation to a new one with the dependent variable $x(u, v)$. In order to do that we need: 
The connections between $\left(x_{u}, x_{v}\right)$ and $\left(u_{x}, u_{y}\right)$. The Jacobi matrix of the change (4) $(x, y) \mapsto(u, v)$ can be written as follows

$$
\frac{\partial(u, v)}{\partial(x, y)}=\left(\begin{array}{cc}
u_{x} & u_{y} \\
0 & 1
\end{array}\right)
$$

The matrix of the inverse change $(u, v) \mapsto(x, y)$ is

$$
\frac{\partial(x, y)}{\partial(u, v)}=\left(\begin{array}{ll}
x_{u} & x_{v} \\
y_{u} & y_{v}
\end{array}\right)=\left(\frac{\partial(u, v)}{\partial(x, y)}\right)^{-1}=\left(\begin{array}{cc}
\frac{1}{u_{x}} & -\frac{u_{y}}{u_{x}} \\
0 & 1
\end{array}\right)
$$

Hence

$$
\left\{\begin{array} { l } 
{ x _ { u } \cdot u _ { x } = 1 } \\
{ x _ { v } \cdot u _ { x } + u _ { y } = 0 }
\end{array} \quad \text { and } \quad \left\{\begin{array}{l}
y_{u}=0 \\
y_{v}=1
\end{array} .\right.\right.
$$

The function $\boldsymbol{x}(u, v)$. Now equation (6) can be transformed into $-x_{v} \cdot u_{x}=f^{\prime}(u) \cdot u_{x}$, hence $x_{v}=-f^{\prime}(u)\left(\right.$ for $\left.u_{x}=z_{x x} \neq 0\right)$ and therefore

$$
x(u, v)=g(u)-v \cdot f^{\prime}(u),
$$

where the function $g(u)$ is an arbitrary function (initial condition) assigning the change $u \mapsto x$ on the $x$-axis: $x(u, 0)=g(u)$; the existence of the change requires $g^{\prime}(u) \neq 0$.

The function $\boldsymbol{z}(u, v)$. Now we can obtain the equation on $z(u, v)$.

$$
u \stackrel{(4)}{=} z_{x}=z_{u} \cdot u_{x}+z_{v} \cdot v_{x} \stackrel{(4)}{=} z_{u} \cdot u_{x}
$$

hence

$$
z_{u}=\frac{u}{u_{x}} \stackrel{(7)}{=} u \cdot x_{u}
$$

On the other hand,

$f(u) \stackrel{(5)}{=} z_{y}=z_{u} \cdot u_{y}+z_{v} \cdot v_{y} \stackrel{(4)}{=} z_{u} \cdot u_{y}+z_{v} \stackrel{(7)}{=} z_{u}\left(-x_{v} \cdot u_{x}\right)+z_{v} \stackrel{(9)}{=}-x_{v} \cdot u+z_{v}$

hence $z_{v}=f+x_{v} \cdot u \stackrel{(8)}{=} f(u)-u \cdot f^{\prime}(u)$. Therefore

$$
z=h(u)+v \cdot\left(f-u \cdot f^{\prime}\right) .
$$

In order to find the function $h(u)$ (depending on $f(u)$ and $g(u)$ since they completely determine the initial Cauchy problem) let us take advantage of (9):

$$
u \cdot x_{u} \stackrel{(9)}{=} z_{u}=h^{\prime}+v \cdot\left(f^{\prime}-f^{\prime}-u \cdot f^{\prime \prime}\right)=h^{\prime}-v \cdot u \cdot f^{\prime \prime}
$$


From (8) $x_{u}=g^{\prime}(u)-v \cdot f^{\prime \prime}(u)$, hence $u \cdot g^{\prime}(u)=h^{\prime}(u)$, and finally

$$
h(u)=\int_{0}^{u} t \cdot g^{\prime}(t) d t=u g(u)-\int_{0}^{u} g(t) d t .
$$

Evaluation of the partial derivatives $z_{x x}, z_{x y}$ and $z_{y y}$ :

$$
\begin{aligned}
& z_{x x} \stackrel{(4)}{=} u_{x} \stackrel{(7)}{=} \frac{1}{x_{u}} \stackrel{(8)}{=} \frac{1}{g^{\prime}-v f^{\prime \prime}} . \\
& z_{x y} \stackrel{(4)}{=} u_{y} \stackrel{(7)}{=}-x_{v} \cdot u_{x} \stackrel{(7)}{=}-\frac{x_{v}}{x_{u}} \stackrel{(8)}{=} \frac{f^{\prime}}{g^{\prime}-v f^{\prime \prime}} . \\
& z_{y y} \stackrel{(5)}{=}[f(u)]_{y}=f^{\prime} \cdot u_{y} \stackrel{(7)}{=}-f^{\prime} \cdot x_{v} \cdot u_{x} \stackrel{(7)}{=}-f^{\prime} \frac{x_{v}}{x_{u}} \stackrel{(8)}{=} \frac{\left(f^{\prime}\right)^{2}}{g^{\prime}-v f^{\prime \prime}} .
\end{aligned}
$$

Initial-value problem. Thus, the function $z(x, y)=z(u(x, y), v(x, y))$ is a solution of (1). It turns out that any solution allows representation (2), i.e., varying $f(u)$ and $g(u)$ (preserving $g^{\prime} \neq 0$ ) we obtain the full set of solutions. Indeed, since $z_{x x} \neq 0$, equation (1) can be represented as $z_{y y}=\frac{z_{x y}^{2}}{z_{x x}}$. Then the initial conditions of the Cauchy problem for this equation can be set along the $x$-axis as two functions in one variable: $z(x, 0)$ and $z_{y}(x, 0)$. As we have seen before on the $x$-axis the variables $x$ and $u$ are interchangeable: $x=g(u)$. Therefore, $z_{x}(x, 0)=u=g^{-1}(x)$; besides that $z_{y}(x, 0) \stackrel{(5)}{=} f(u)=f\left(g^{-1}(x)\right)$. Altogether:

$$
\begin{aligned}
z(x, 0) & =\int_{0}^{x} g^{-1}(t) d t \in C^{2}, \\
z_{y}(x, 0) & =f\left(g^{-1}(x)\right) \in C^{1} .
\end{aligned}
$$

Thus, the pairs of functions $f(u), g(u)$ and $z(x, 0), z_{y}(x, 0)$ are interchangeable which means system (2) gives the general solution of (1).

Smoothness of the initial conditions $f(u)$ and $g(u)$. As one can see from (3), for the existence of $z_{x x}$ it is necessary that $g(u) \in C^{1}$ and $f(u) \in C^{2}$. At the same time these conditions are sufficient for the existence of $z_{x x}, z_{x y}$ and $z_{y y}$.

\section{Domain of the solution. Caustic}

The surface $F \subset E^{3}$ as the domain of the solution. The change of variables $(x, y) \mapsto(u, v)(4)$ is not accidental in the least. The matter is that the natural domain for the solution of (1) is the surface $F \subset E^{3}$ with the radius vector $(x(u, v), y(u, v), z(u, v))$ rather than the $x y$-plane. The surface $F$ is rectilinear:

$$
\left(\begin{array}{l}
x \\
y \\
z
\end{array}\right)=a(u)+v \cdot b(u) \quad \text { with } \quad a(u)=\left(\begin{array}{c}
g \\
0 \\
u g-\int g
\end{array}\right), \quad b(u)=\left(\begin{array}{c}
-f^{\prime} \\
1 \\
f-u f^{\prime}
\end{array}\right) \text {. }
$$


Moreover, it is a torse (i.e., a rectilinear surface with tangent plane stable along the generators), a characteristic property of which is the condition $\dot{b} \in \operatorname{span}\{\dot{a}, b\}$ ([Sp, p. 284] or [U3]). Indeed, $\dot{b}=-\frac{f^{\prime \prime}}{g^{\prime}} \dot{a}$.

Let us notice that the surface $F$ is $C^{2}$ smooth (because in the cartesian parametrization $(x, y, z(x, y))$ the function $z(x, y)$ is $\left.C^{2}\right)$ despite the $C^{1}$ smoothness of the parametrization given by (11). Such a drop in smoothness for this more "geometrical" parametrization is a common occurrence - see Theorem 1 [U4].

There are no planar points on the surface $F$ (due to $z_{x x} \neq 0$ ) and consequently it consists of the parts of cylinders, cones and tangent developables glued pairwise along their rectilinear generators [U1].

Singularities. If we assign the initial conditions $f(u)$ and $g(u)$ defined on the segment $\left[u_{1}, u_{2}\right]$ of the $x$-axis (to be more precise on the segment $\left[x_{1}, x_{2}\right]$ with $\left.x_{1}=g\left(u_{1}\right), x_{2}=g\left(u_{2}\right)\right)$ then the equations (2) give the solution on the whole strip $(u, v) \in\left[u_{1}, u_{2}\right] \times(-\infty, \infty)$. Thus one can say, the solution is naturally extended to infinity along the generators of the surface.

Yet, in the general case on every generator there is a singular point at which the tangent plane to $F$ degenerates into a line. Indeed, the tangent plane of the surface $a(u)+v \cdot b(u)$ is spanned by the basis tangent vectors $\dot{a}+v \cdot \dot{b}$ and $b$ :

$$
\dot{a}+v \dot{b}=\left(g^{\prime}-v f^{\prime \prime}\right)\left(\begin{array}{l}
1 \\
0 \\
u
\end{array}\right), \quad b=\left(\begin{array}{c}
-f^{\prime} \\
1 \\
f-u f^{\prime}
\end{array}\right)
$$

and the degeneracy condition, $\operatorname{rank}\{\dot{a}+v \dot{b}, b\}<2$, is equivalent to

$$
g^{\prime}(u)-v f^{\prime \prime}(u)=0 .
$$

Thus on the generator $u_{0}$ all points except $v=g^{\prime}\left(u_{0}\right) / f^{\prime \prime}\left(u_{0}\right)$ have non-degenerate tangent planes.

Cylinders. If we want to have no singularities in the strip $\left[u_{1}, u_{2}\right] \times(-\infty, \infty)$ we must require $f^{\prime \prime}(u) \equiv 0$, i.e., $f(u)=\alpha u+\beta$ with constant $\alpha$ and $\beta$. Then the direction of the generators $b(u)=(-\alpha, 1, \beta)$ is constant (see (11)); the surface $F$ is a cylinder $a(u) \times b \subset E^{3}$.

The last statement in fact is equivalent to the well-known Pogorelov's theorem: a complete surface of vanishing Gaussian curvature is a cylinder [P, p. 696] or [Sp, pp. 363-367] (true, in that theorem planar points were also taken into consideration, but our treatment could be extended to cover them too - see Section 3).

The singularities found are preserved under the projection of the surface $F$ onto the $x y$-plane (that will be discussed further). Therefore the last statement can be reformulated as follows: the solution of equation (1) is defined and regular over the whole $x y$-plane if and only if the initial function $f(u)$ is linear. 
Now we shall assume that there are no points with $f^{\prime \prime}=0$ on the segment $\left[u_{1}, u_{2}\right]$. Then equation (12) specifies the singularity curve

$$
a^{*}(u)=a(u)+\frac{g^{\prime}}{f^{\prime \prime}}(u) \cdot b(u)
$$

on the strip $\left[u_{1}, u_{2}\right] \times(-\infty, \infty)$ of the surface $F$. In order to understand the general picture we impose two additional assumptions.

a) Let us suppose the function $g^{\prime} / f^{\prime \prime}$ to be $C^{1}$ smooth. Then the singularity curve (13) is also $C^{1}$ smooth and its tangent vector is $\dot{a}^{*}=\left(g^{\prime} / f^{\prime \prime}\right)^{\prime} \cdot b$.

b) We will assume that, on the segment considered, either $\left(g^{\prime} / f^{\prime \prime}\right)^{\prime} \equiv 0$ or $\left(g^{\prime} / f^{\prime \prime}\right)^{\prime}$ is never zero.

Cones. Let $\left(g^{\prime} / f^{\prime \prime}\right)^{\prime} \equiv 0$. Then $\dot{a}^{*} \equiv 0$ and the curve (13) degenerates into a point: $a^{*}(u)=a_{0}^{*}$. All the generators of the surface $F$ pass through that point. Under the projection onto the $x y$-plane the picture is preserved: all the generators in the plane pass through some fixed point $P_{0}$, the strip $\left[u_{1}, u_{2}\right] \times(-\infty, \infty)$ is projected onto the interior of two vertical angles between the straight lines $\left(x\left(u_{1}\right), P_{0}\right)$ and $\left(x\left(u_{2}\right), P_{0}\right)$.

Tangent developables. Let us now consider the case $\left(g^{\prime} / f^{\prime \prime}\right)^{\prime} \neq 0$ everywhere on $\left[u_{1}, u_{2}\right]$. In this case the singularity curve (13) is a genuine curve which envelopes the generators of the surface $F$. Such a surface is called a tangent developable and the curve itself is called the edge of regression. A general idea of what a tangent developable looks like can be obtained from a drawing in [Sp, p. 208]. After the projection of $F$ onto the $x y$-plane, the edge of regression (13) turns into a curve called the caustic of the solution (2). The generators $u=$ const in the $x y$-plane are tangent to the caustic, each generator at its own point. The caustic is a convex curve since its tangent vector $\tau(u)$ (which is the projection of $b(u))$ is $\left(-f^{\prime}, 1\right)$ and the frame $\{\tau, \dot{\tau}\}$ keeps its orientation as long as $f^{\prime \prime} \neq 0$. Comparing (12) and (3) we notice that while approaching the caustic the Hesse matrix goes to infinity.

Every generator is divided by the caustic point into two parts; correspondingly, the domain of the solution (2) is decomposed into the parts consisting of the points of the "bottom rays" and "top rays" of the generators. The foregoing can be illustrated as follows.

Example. Let the functions $f(u)=|u|^{3}$ and $g(u)=u$ be given on the whole $x$-axis. Then the surface $F$ is

$$
\left(\begin{array}{l}
x \\
y \\
z
\end{array}\right)=\left(\begin{array}{c}
u \\
0 \\
\frac{u^{2}}{2}
\end{array}\right)+v \cdot\left(\begin{array}{c}
-3 u|u| \\
1 \\
-2|u|^{3}
\end{array}\right) .
$$

The equation of the caustic (12) in the $u v$-plane is $6|u| v=1$. In the $x y$-plane the caustic is as follows:

$$
\left(\begin{array}{l}
x \\
y
\end{array}\right)=\left(\begin{array}{l}
g \\
0
\end{array}\right)+v(u) \cdot\left(\begin{array}{c}
-f^{\prime} \\
1
\end{array}\right)=\left(\begin{array}{c}
u / 2 \\
\frac{1}{6|u|}
\end{array}\right)
$$


hence, eliminating $u$, we get

$$
12|x| y=1,
$$

i.e., the caustic consists of two branches of hyperbolae:

$$
y= \begin{cases}\frac{1}{12 x}, & \text { for } x>0 \\ -\frac{1}{12 x}, & \text { for } x<0\end{cases}
$$

When $x$ approaches zero, the caustic goes to infinity and this is to be expected since $f^{\prime \prime}=6|u| \rightarrow 0$. There are three different branches of the solution $z(x, y)$.

Branch 1. This is defined at the points of generators which are below the caustic, on "bottom rays", i.e., whenever $y<\frac{1}{12|x|}$. The map $(u, v) \mapsto(x, y)$

$$
\left\{\begin{array}{l}
x=u-3 v \cdot u|u| \\
y=v
\end{array}\right.
$$

can be inverted in this domain:

$$
\left\{\begin{array}{l}
u=\frac{2 x}{1+\sqrt{\psi_{1}}}, \quad \text { where } \psi_{1}=1-12|x| \cdot y . \\
v=y
\end{array}\right.
$$

The solution $z(x, y)=\frac{2 x^{2}}{3}\left(1-\frac{\psi_{1}}{\left(1+\sqrt{\psi_{1}}\right)^{2}}\right)$.

Branch 2. This consists of the points of the "top rays" tangent to the right hyperbola. In this case $u>0$, the branch is defined in the whole Quadrant II and in Quadrant I below the hyperbola $y=\frac{1}{12 x}$. The inversion of (15) is

$$
\left\{\begin{array}{l}
u=\frac{2 x}{1-\sqrt{\psi_{2}}}, \quad \text { where } \psi_{2}=1-12 x \cdot y, \\
v=y
\end{array}\right.
$$

and the function $z(x, y)=\frac{2 x^{2}}{3}\left(1-\frac{\psi_{2}}{\left(1-\sqrt{\psi_{2}}\right)^{2}}\right)$.

Branch 3. This consists of the points of "top rays" tangent to the left hyperbola. In this case $u<0$, the branch is defined in the whole Quadrant I and in Quadrant II below the hyperbola $y=-\frac{1}{12 x}$. The inversion of (15) is

$$
\left\{\begin{array}{l}
u=\frac{2 x}{1-\sqrt{\psi_{3}}}, \quad \text { where } \psi_{3}=1+12 x \cdot y, \\
v=y
\end{array}\right.
$$

and the function $z(x, y)=\frac{2 x^{2}}{3}\left(1-\frac{\psi_{3}}{\left(1-\sqrt{\psi_{3}}\right)^{2}}\right)$. 


\section{Points with $z_{x x}=0$}

In this Section we discuss the extension of Theorem 1 removing the assumption $z_{x x} \neq 0$. The points with $z_{x x}=0$ are naturally split into two classes: parabolic and planar points.

Parabolic points: $z_{x x}=z_{x y}=0, z_{y y} \neq 0$ The necessity for individual consideration of these points (they are not in the solution (2) - see (3)) is entirely generated by the choice of the coordinate system. If we swap the axes $x$ and $y$ then the points $z_{y y}=z_{x y}=0, z_{x x} \neq 0$ (which are currently in the solution (2)) will be found in the same disadvantageous position.

It can be easily seen from (3) and by interchanging $x$ and $y$ that the parabolic points with $z_{x x}=0$ always lie on a generator parallel to the $x$-axis. Such a generator cannot intersect the $x$-axis (unless it coincides with the whole $x$-axis) and therefore setting the initial values for (2) along the $x$-axis, we automatically exclude the points in question from consideration.

Changing the locus of initial values from the $x$-axis to an arbitrary (even space) curve we can include the points discussed in the solution but lose the elegance of formulae (2).

Planar points: $z_{x x}=z_{x y}=z_{y y}=0$ These points in turn are split into two classes:

(1) planar points possessing planar neighbourhood;

(2) planar points being a limit of parabolic points.

Points which are planar together with the vicinity lie on a flat piece of the surface $F$; the function $z$ in that vicinity is linear.

The planar points of type (2) inherit the rectilinear structure from close parabolic points: these points fill the whole generator which is the limiting position of the corresponding parabolic generators (this statement is implied by the following easy-to-check fact: if a generator of the surface $F$ has a parabolic point then all the points of that generator are parabolic; a detailed discussion and bibliography can be found in [U2]).

Therefore, the surface $F$ without flat pieces is rectilinear; some generators are parabolic, the rest are planar. The parabolic generators make up an open set; the planar generators form a closed one. The latter may have a rather complicated structure: for instance, they might cut out the Cantor set on a trajectory, transversal to the generators.

The planar points of type (2) possess another interesting property. Despite the $C^{2}$ smoothness of the surface $F$, the vector field assigning the directions of the generators (and its projection onto the $x y$-plane) is $C^{1}$ smooth when we allow only the planar points are present parabolic points and is only $C^{0}$ smooth. Such an effect may appear even in the case of a single planar generator [U2]. 


\section{References}

[CH] R. Courant and D. Hilbert, Methods of Mathematical Physics, Vol. II: Partial Differential Equations by R. Courant, Interscience publishers 1962.

[HW] Ph. Hartman and A. Wintner, On the asymptotic curves of a surface, Amer. J. Math. 73 (1951), 149-172.

[J1] K. Jörgens, Über die Lösungen der Differentialgleichung $r t-s^{2}=1$, Math. Ann. 127 (1954), 130-134. (German)

[J2] K. Jörgens, Harmonische Abbildungen und die Differentialgleichung $r t-s^{2}=1$, Math. Ann. 129 (1955), 330-334. (German)

[P] A. V. Pogorelov, Exterior geometry of convex surfaces. Nauka, Moscow 1969. (Russian)

[Sp] M. Spivak, A Comprehensive Introduction to Differential Geometry, Vol. III, Publish or Perish, Inc., Berkeley 1979, p. 466.

[SW] F. Schulz and L. Wang, Isolated singularities of Monge-Ampère equations, Proc. Amer. Math. Soc. 123 (1955), 3705-3708.

[U1] V. Ushakov, Riemannian manifolds and surfaces of constant nullity. Thesis, St.-Petersburg University 1993. (Russian)

[U2] V. Ushakov, Parametrization of developable surfaces by asymptotic lines, Bull. Austral. Math. Soc. 54 (1996), 411-421.

[U3] V. Ushakov, Smoothness of the solution of trivial Monge-Ampère equation, Bull. Austral. Math. Soc. 56 (1997), 439-445.

[U4] V. Ushakov, Smoothness of tangent developable, preprint, the University of Melbourne 1996.

Vitaly Ushakov

8/11 Tiuna Gve.

Elwood, 3184

Vic, Australia

e-mail:vit@ms.unimelb.edu.au

(Received: January 5, 1998) 